\title{
Evaluation of cardiac function by global longitudinal strain before and after treatment with sofosbuvir-based regimens in HCV infected patients
}

Maria Mazzitelli ${ }^{1 \dagger}$, Carlo Torti ${ }^{1{ }^{*}}$, Jolanda Sabatino ${ }^{2}$, Greta Luana D'Ascoli ${ }^{2}$, Chiara Costa ${ }^{1}$, Vincenzo Pisani ${ }^{1}$, Elena Raffetti ${ }^{3}$, Salvatore De Rosa ${ }^{2}$, Alessio Strazzulla ${ }^{1}$, Alfredo Focà ${ }^{4}$, Maria Carla Liberto ${ }^{4}$, Ciro Indolfi ${ }^{2}$ and the CARDIAC study group

\begin{abstract}
Background: Possible cardiotoxicity of sofosbuvir in humans has not been demonstrated yet. Also, since HCV can exert deleterious effects on hearth function, it is of interest to know whether HCV eradication provides any benefits using global longitudinal strain (GLS), a measure of left ventricular function more reliable than ejection fraction (EF).

Methods: Patients eligible for treatment with the combination therapy for HCV were invited to perform a transthoracic cardiac ultrasound at four different time points: before starting treatment, after one month, at the end of treatment and, after six month. Left ventricular function was measured with both EF and GLS.

Results: From March 2015 to December 2016, 82 patients were enrolled. Fifty-six percent patients were males. Mean age was 66.12 (SD: 9.25) years. About 20\% patients did not present any cardiovascular risk factors or comorbidities. A worsening trend of GLS was observed. Variations were not found to be statistically significant when EF was studied along the follow-up. However, when GLS was studied, its variations were found to be statistically significant indicating a worsening effect, albeit with different trends in patients who underwent treatment for three months compared to six months. Worsening of GLS was found to be statistically significant even after adjusting for body mass index and liver fibrosis, independently from treatment duration.
\end{abstract}

Conclusions: Our results showed unexpected worsening of left ventricular function when measured through GLS after HCV treatment response induced by DAAs including sofosbuvir. Although this result is not proven to be clinically significant, the safety profile of sofosbuvir-based regimens needs to be studied further.

Keywords: Cardiac function, HCV eradication, DAA treatment, Longitudinal study

\section{Background}

Extra-hepatic manifestations (such as neoplastic, autoimmune and vascular diseases) occur in about $70 \%$ of patients infected by hepatitis $\mathrm{C}$ virus (HCV) [1-3]. Among these manifestations, cardiovascular diseases (CVD) are more prevalent in HCV infected patients, but

\footnotetext{
* Correspondence: torti.carlo@libero.it

${ }^{+}$Maria Mazzitelli and Carlo Torti contributed equally to this work.

'Unit of Infectious and Tropical Diseases, Department of Medical and

Surgical Sciences, "Magna Graecia" University of Catanzaro, Viale Europa, 88100 Catanzaro, Italy

Full list of author information is available at the end of the article
}

mechanisms are currently unknown. HCV related inflammation, oxidative stress $[4,5]$ and direct damage due to $\mathrm{HCV}$ infecting cardiac cells [6-8] might have a impact.

Animal studies reported death for cardiac causes after administration of a sofosbuvir metabolite at blood concentrations much higher than the therapeutic index used in humans [9]. Currently there is a lack data on the effects of sofosbuvir on heart function. Such data are important to confirm safety of sofosbuvir because we are currently treating aging populations with a significant prevalence of heart diseases. On the other way round, it is possible that

(c) The Author(s). 2018 Open Access This article is distributed under the terms of the Creative Commons Attribution 4.0 International License (http://creativecommons.org/licenses/by/4.0/), which permits unrestricted use, distribution, and reproduction in any medium, provided you give appropriate credit to the original author(s) and the source, provide a link to the Creative Commons license, and indicate if changes were made. The Creative Commons Public Domain Dedication waiver (http://creativecommons.org/publicdomain/zero/1.0/) applies to the data made available in this article, unless otherwise stated. 
clearance of $\mathrm{HCV}$ with interferon-free regimens would act favourably, as it was previously demonstrated that $\mathrm{HCV}$ eradication with interferon based regimens is able to reduce mortality for cardiovascular events [10,11].

Left ventricular function (LVF) is routinely evaluated through the ejection fraction (EF), calculated by means of the modified Simpson method in current clinical practice, with the use of trans-thoracic echocardiography [12]. Trans-thoracic echocardiography based speckle tracking assessment is more reliable and precise for the assessment of myocardial function than trans-oesophageal ultrasound [13].

More recently, the global longitudinal strain (GLS) was developed as a more reliable index to measure left ventricular function [14]. Indeed, GLS was shown to be a valuable clinical parameter and a independent predictor of all cause mortality in patients with CVD [15]. Moreover, variations of GLS have been found in diverse conditions such as doxorubicin-induced cardiomyopathy, HIV infection in children and young adults, or viral myocarditis [16-19]. In these conditions, even minor variations of GLS were clinically meaningful, even when EF seemed to be preserved [18, 19]. For instance, data showed that GLS provides incremental diagnostic and prognostic information, that are correlated with histological findings in patients with viral myocarditis for whom conventional 2D echocardiography is unspecific, particularly in those with a preserved EF $[18,19]$. This correlation was independent from conventional 2D echocardio-graphic parameters showing that strain rate and strain imaging are more sensitive in the detection of early changes or mild myocardial damage. Moreover, patients with impaired strain rate and strain at the acute phase of the disease showed worse short-time echocardiographic outcomes. For these patients, clinical history, physical examination, ECG, and serology were shown to be unreliable compared with GLS.

\section{Methods}

\section{Aim}

In the present study, we aimed at measuring possible changes of cardiovascular function in patients with chronic HCV infection before and after sofosbuvir-based regimens, using both left ventricular EF and GLS. The latter was chosen as advanced biomarker to measure the effect.

\section{Population and data collection}

We conducted a longitudinal study from March 2015 to January 2017, enrolling all HCV infected patients treated with sofosbuvir-based regimens at the outpatient clinic of "Mater Domini" teaching hospital in Catanzaro (Italy), according to the criteria set by the Italian Medicinal Agency (AIFA) (see Additional file 1: Table S1). For patients without clinical cirrhosis or extra-hepatic manifestations, transient elastography (FibroScan ${ }^{\mathrm{Tu}}$ ) was performed in order to estimate liver fibrosis so as to ascertain indications for treatment.

Exclusion criteria were: age less than 18 years old, pregnancy, and severe chronic disease (estimated glomerular filtration rate, eGFR $<30 \mathrm{~mL} / \mathrm{min}$ ).

Patients were assessed at four time points: baseline (i.e., before treatment initiation), after one month, at the end of the treatment course (either month 3 or month 6), and after 6 months from the end of treatment (off treatment follow-up).

Cardiac ultrasound was performed at baseline and at each follow-up using trans-thoracic Vivid E9 ultrasound. Speckle tracking echocardiography analysis was performed from apical views. Standard grayscale 2D images were obtained at a frame rate of 70-90 frames/s during three cardiac cycles and software package (EchoPAC ${ }^{\mathrm{mm}}$, GE healthcare) was used for offline analysis. Two expert cardiologists (L.G.D.A. and J.S.) performed cardiac ultrasound blinded of previous examinations, type and length of prescribed treatments.

At baseline, risk factors for CVD (i.e., hypertension, diabetes mellitus, cigarette smoking, previous stroke or myocardial infarction were recorded), and heart diseases were carefully investigated. Patients were considered to be underweighted $\left(\mathrm{BMI} \leq 18.4 \mathrm{Kg} / \mathrm{m}^{2}\right)$, normal $(\mathrm{BMI}=18.5-$ $\left.24.9 \mathrm{Kg} / \mathrm{m}^{2}\right)$, over-weighted $\left(\mathrm{BMI}=25-29.9 \mathrm{Kg} / \mathrm{m}^{2}\right)$ or obese $\left(\mathrm{BMI} \geq 30 \mathrm{Kg} / \mathrm{m}^{2}\right)$ [20].

Complete blood count, AST, ALT, total and fractioned bilirubin, and HCV RNA were recorded at enrolment and each follow-up points. Indirect indices of fibrosis, such as Fibrosis 4 index (FIB-4) and AST to platelets ratio (APRI) score were calculated at baseline and at month 6 after the end of treatment [21-23]. At these time points, alpha-fetoprotein, cholesterol, creatinine, glucose, INR, triglycerides were also evaluated. Data were stored in an ad-hoc electronic database.

Drug interactions with other co-medications were carefully evaluated using the application HEP Drug Interaction [24]. Drugs with a significant risk of interaction with antivirals were substituted. For example, after cardiological consultation, amlodipine was reduced from $10 \mathrm{mg}$ to $5 \mathrm{mg}$ per day in patients who received daclatasvir or ledipasvir, if possible, or otherwise substituted.

This study was coordinated by the Infectious and Tropical Diseases Unit in collaboration with the Cardiology Unit of "Mater Domini" teaching hospital in Catanzaro (Italy) and was conducted in accordance with the guidelines of the Declaration of Helsinki and the principles of Good Clinical Practice [25-27]. The local Ethical Committee (Calabria Region) approved the study protocol and written informed consent was obtained from all subjects enrolled. 


\section{Statistical analysis}

To adjust the analysis for treatment duration, the enrolled patients were ranked into two groups: group A, i.e. patients with indication for a 3 month treatment, and group B, i.e. patients with indication for a 6 month treatment with DAAs. Study parameters were expressed as means (standard deviation, SD) or proportions as appropriate. FIB-4, APRI score, alpha-fetoprotein, creatinine, cholesterol, glucose, haemoglobin, and triglycerides values at baseline were compared with those at last follow-up using Student's t-test for paired data. We evaluated the temporal trends of AST, ALT, platelet count, total bilirubin, EF and GLS using univariate mixed models for repeated measures. We also assessed the temporal trend of GLS using a multivariate mixed model adjusting for BMI, fibrosis and duration of treatment (3 or 6 months). Moreover, although in the analysis hypertension was not a confounder by definition, since it could have been associated with the outcome (GLS) but not with the exposure, we tested whether hypertension was a effect modifier.

Lastly we explored whether ribavirin could have a role on the change of GLS over time using a mixed model with an interaction term between ribavirin and time.

All statistical tests were two-sided, assuming a level of significance of 0.05 and were performed using Stata software version 12.0 (StataCorp, College Station, TX, USA).

\section{Results}

\section{Patient flow and characteristics}

Among 109 patients who started a DAA treatment during the study period, 87 subjects were eligible and 82 were enrolled (56\% males, mean age of 66.1 years). Amongst these patients, 71/82 (86.6\%) continued follow-up until the end of the study (Fig. 1).

Seventy-two (87.9\%) patients met AIFA criterion 1 or 4. Nine patients had extra-hepatic manifestations (AIFA criterion 3), and one patient had a HCV RNA relapse after liver transplantation (AIFA criterion 2). Fifty-seven $(69.5 \%)$ patients were prescribed a treatment lasting for three months, while 25 (30.5\%) were prescribed a treatment for six months. The baseline characteristics of patients are described in Table 1. With regards to $\mathrm{CV}$ risk factors, $59.3 \%$ patients were overweighed, $4.7 \%$ had a previous major cardiovascular event (stroke or myocardial infarction), $17.4 \%$ were smokers, $62.8 \%$ had hypertension and $26.7 \%$ had diabetes mellitus. Overall, only $20 \%$ of subjects did not present any CV risk factors or comorbidities. About 70\% patients had previous experience to interferon-based regimens.

\section{Treatment course}

Prescribed treatments and related outcomes are described in Table 2. Most patients (97.56\%) reached the end of treatment; only one patient stopped prematurely for a psychotic syndrome and another for virological failure. Seventy-nine (96.4\%) patients gained sustained virological response (SVR) at weeks 12 after the end of treatment. Two patients $(2.4 \%)$ had virological failure. All patients tolerated treatments very well, without any severe adverse events recorded. Among 49 patients who received ribavirin, folic acid and/or erythropoietin were added for anaemia in $11(22.4 \%)$ and in $1 / 49(2.04 \%)$ ribavirin was stopped for the same reason. Table 3 shows temporal trends of selected parameters. Liver parameters improved, whereas cholesterol rose in both groups (treatment length of 3 or 6 months).

\section{Evaluation of cardiac function}

At baseline, mean EF and GLS were $56.7 \%$ and $-20.9 \%$, respectively. Hence, 20/82 (24.1\%) patients had abnormal EF $(<55 \%)$, while $3 / 82(3.6 \%)$ had abnormal GLS $(>-16.5 \%)$ according to litereature standards [28, 29]. Compared to those with lower values, subjects with GLS $\geq$ median value of the study population $(-20.3 \%)$ had higher BMI (mean 27.9 vs. 26.0), higher haemoglobin (14.6 vs. $13.5 \mathrm{~g} / \mathrm{dL}$ ), higher triglycerides ( 123.5 vs. $98.3 \mathrm{mg} / \mathrm{dL})$ and a greater proportion of current smokers was found (71.4\% vs. $44.1 \%)$ (see, Additional file 1: Table S2). Moderate mitral and tricuspid insufficiencies were diagnosed in 2 patients.

As illustrated in Table 3, while there were not statistically significant variations of EF along the follow-up in both groups, a statistically significant worsening of GLS was found in the group of patients treated for three months (group A), while in patients treated for six months (group B) only a tendency towards a statistically significant worsening was found. Interestingly, GLS displayed a biphasic trend in the 3-month group, decreasing from $-20.8 \%$ at baseline to $-21.4 \%$ at month 1 , before rising up to $-20.3 \%$ at the end of the follow-up ( $p=0.031)$ (Fig. 2A). By contrast, GLS increased steadily from $-21.1 \%$ to $-20.1 \%$ in the six-month group $(p=0.097)$ (Fig. 2B). The rise of GLS over time was confirmed in a multivariate mixed model adjusted for BMI, liver fibrosis and treatment length with a mean GLS increase of $0.07(0.01-0.13)$ per month $(p=0.013)$ (see, Additional file 1: Table S3).

Lastly, we explored whether hypertension was a effect modifier but we did not found any significant evidences (coefficient $-0.43,95 \% \mathrm{CI}$ : -1.42 to $0.55 ; p=0.388$ ). We also tested whether ribavirin could have a role on the change of GLS over time and in a mixed model with an interaction term between ribavirin and time but ribavirin exposure did not exert a statistically significant effect on GLS (absent ribavirin coefficient - 0.607, 95\% CI: -1.608 to $0.395 ; p<0.235$ ) or a statistically significant role as effect modifier was not demonstrated (coefficient -0.018 , 95\% CI: -1.69 to $0.39 ; p=0.749$ ). 


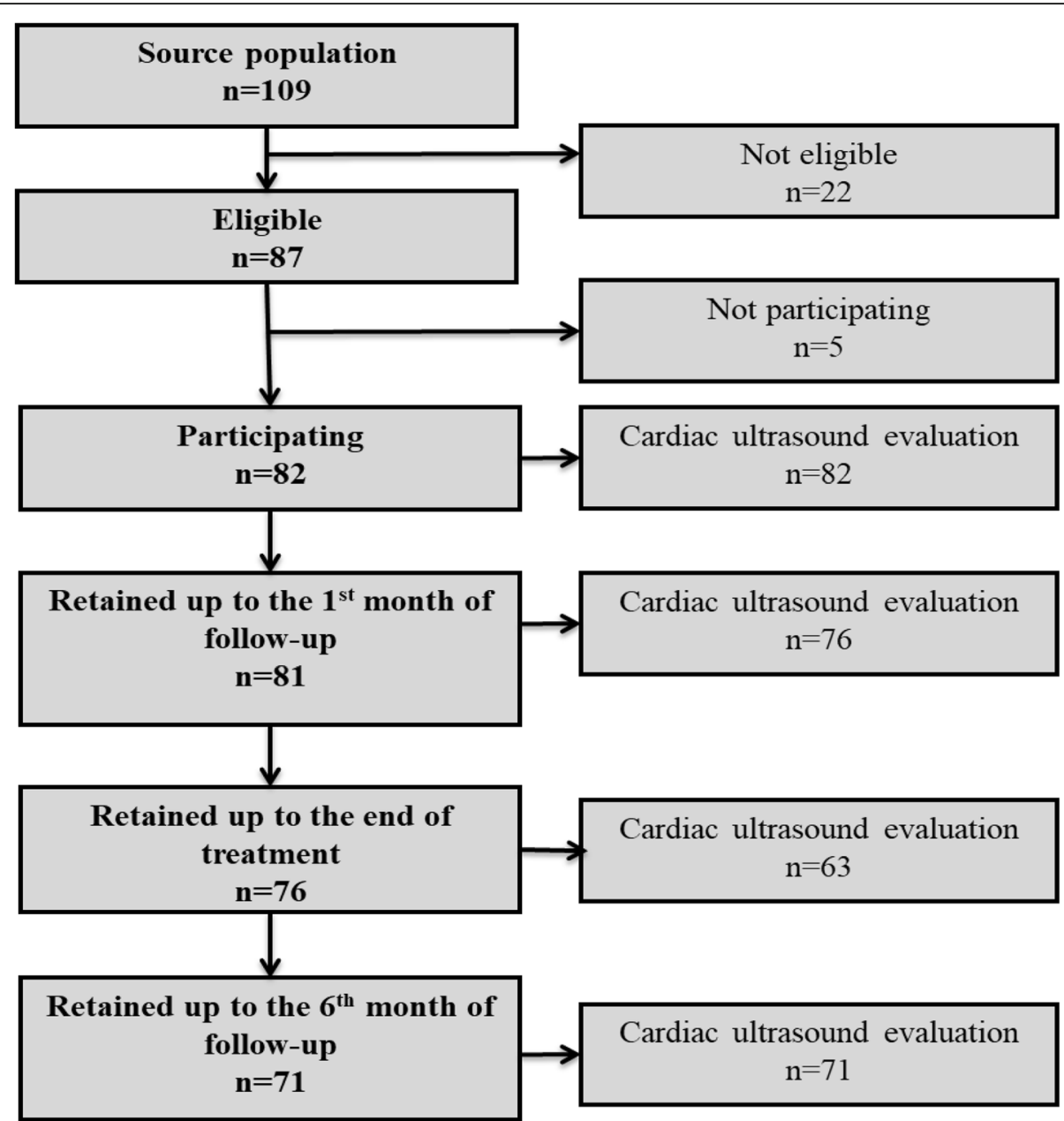

Fig. 1 Flow chart of the patients along the study time points. Eighty-two patients decided to participate and underwent cardiac ultrasound at baseline. Fifty-seven patients were prescribed a 3-month treatment (group A) while 25 patients underwent treatment for 6 months (group B). Among patients who showed up at clinical checks, 76/81 presented to perform cardiac ultrasound at first month, 63/76 at the end of treatment and $71 / 71$ at the last follow-up point

\section{Discussion}

The main finding of our study is that cardiac function measured through GLS seemed to worsen in the overall population, while EF did not change significantly. This may indicate that sofosbuvir based treatment could exert a negative impact on cardiac function. Possible toxicity of sofosbuvir may be supported from data showing that development of another NS5B polymerase inhibitor (BMS5986094) was stopped after a safety signal of cardiotoxicity [30]. In this work a young male died for rapidly progressive heart failure and $41.2 \%(14 / 34)$ patients had some evidence of cardiac dysfunction (6/14 with $\mathrm{EF}<30 \%$ and $8 / 14$ from 30 to $50 \%$ ). So, as far as cardiotoxicity is concerned, a class effect of NS5B polymerase inhibitors should be studied further. Interestingly, after stopping DAAs, GLS continued to worsen, possibly indicating a prolonged effect. In addition, since
EF remained stable, we may hypothesize that, similar to other conditions [14, 15], GLS is a more sensitive method to measure cardiac function.

However, the clinical significance and the long-term effects of the GLS variations in our patients are unknown. Correlations with other biomarkers of heart dysfunction (such as troponin, NT-pro-BNP, and micro-RNAs) [3134] and long term studies with "hard" clinical end-points would be helpful. Also, it is difficult to explain why such an effect was demonstrated. In fact, besides a direct effect of sofosbuvir, other explanations may be found, including a random effect due to the small number of patients, an effect of concomitant drugs, or an indirect effect of $\mathrm{HCV}$ eradication mediated by inflammatory changes $[4,35]$. So, the major difficulty that comes with the dataset studied herein is to dissect whether the effect can be ascribed entirely to sofosbuvir or to other factors. For this reason, more 
Table 1 Characteristics of the enrolled patients overall and by length of treatment (group A: treatment lasting for 3 months and group B: treatment lasting for 6 months)

\begin{tabular}{|c|c|c|c|c|}
\hline Variable & Total n (\%) & $\begin{array}{l}\text { Group A } \\
\mathrm{n}(\%)\end{array}$ & $\begin{array}{l}\text { Group B } \\
\mathrm{n}(\%)\end{array}$ & $p$-value \\
\hline \multicolumn{5}{|l|}{ Gender } \\
\hline Male & $46(56.1)$ & $35(61.4)$ & $11(44)$ & \\
\hline Female & $36(43.9)$ & $22(38.6)$ & $14(56)$ & 0.144 \\
\hline \multicolumn{5}{|l|}{ Age (years) } \\
\hline$\leq 60$ & $22(26.8)$ & $17(29.8)$ & $5(20)$ & \\
\hline $61-68$ & $22(26.8)$ & $16(28.1)$ & $6(24)$ & \\
\hline $69-74$ & $24(29.3)$ & $15(26.3)$ & $8(32)$ & \\
\hline$\geq 75$ & $14(17.1)$ & $9(15.8)$ & $6(24)$ & 0.666 \\
\hline \multicolumn{5}{|l|}{ Type of liver disease } \\
\hline No cirrhosis or HCC & $45(54.9)$ & $34(59.6)$ & $9(36)$ & \\
\hline Cirrhosis & $35(42.7)$ & $23(40.4)$ & $14(56)$ & \\
\hline HCC with cirrhosis & $2(2.4)$ & $0(0)$ & $2(8)$ & 0.025 \\
\hline \multicolumn{5}{|l|}{ Transient elastography } \\
\hline None & $7(8.5)$ & & & \\
\hline F0 & $0(0)$ & $0(0)$ & $0(0)$ & \\
\hline F1 & $2(2.4)$ & $2(3.9)$ & $0(0)$ & \\
\hline F2 & $3(3.8)$ & $3(5.9)$ & $0(0)$ & \\
\hline F3 & $33(40.2)$ & $29(56.9)$ & $3(13)$ & \\
\hline F4 & $37(45.1)$ & $17(33.3)$ & $20(87)$ & $<0.01$ \\
\hline
\end{tabular}

HCV Genotype

\begin{tabular}{|c|c|c|c|c|}
\hline $1 a$ & $2(2.4)$ & $1(1.7)$ & $1(4)$ & \\
\hline $1 b$ & $60(73.3)$ & $42(73.7)$ & $17(68)$ & \\
\hline 2 & $6(7.3)$ & $3(5.3)$ & $3(12)$ & \\
\hline $2 a / 2 c$ & $5(6.1)$ & $6(10.5)$ & $0(0)$ & \\
\hline 3 & $4(4.8)$ & $1(1.7)$ & $3(12)$ & \\
\hline 4 & $5(6.1)$ & $4(7.1)$ & $1(4)$ & 0.148 \\
\hline \multicolumn{5}{|l|}{ Co-infections } \\
\hline None & $81(98.8)$ & $56(98.3)$ & $25(100)$ & \\
\hline HIV + & $1(1.2)$ & $1(1.7)$ & $0(0)$ & \\
\hline HBsAg +/HIV - & $0(0)$ & $0(0)$ & (0) & 0.505 \\
\hline \multicolumn{5}{|l|}{ BMl } \\
\hline Normal & $23(28)$ & $18(31.6)$ & $5(20)$ & \\
\hline Overweight/Obese & $59(71.0)$ & $39(68.4)$ & $20(80)$ & 0.283 \\
\hline \multicolumn{5}{|c|}{ Risk factors for $C V$ diseases ${ }^{a}$} \\
\hline None & $19(23.2)$ & $15(26.3)$ & $4(16)$ & 0.308 \\
\hline Hypertension & $51(62.2)$ & $34(59.6)$ & $17(68)$ & 0.473 \\
\hline Diabetes mellitus & $21(25.6)$ & $12(21.1)$ & $8(32)$ & 0.288 \\
\hline Smoking habits & $14(17.1)$ & $11(19.3)$ & $3(12)$ & 0.419 \\
\hline Previous CV events & $4(4.8)$ & $3(5.3)$ & $1(4)$ & 0.807 \\
\hline \multicolumn{5}{|l|}{ Comorbidities $^{a}$} \\
\hline None & $19(23.2)$ & $15(26.3)$ & $4(16)$ & 0.308 \\
\hline $\mathrm{eGFR}<90 \mathrm{ml} / \mathrm{min}$ & $43(52.4)$ & $32(39)$ & $11(13.4)$ & 0.350 \\
\hline
\end{tabular}

Table 1 Characteristics of the enrolled patients overall and by length of treatment (group A: treatment lasting for 3 months and group B: treatment lasting for 6 months) (Continued)

\begin{tabular}{lllll}
\hline Variable & Total n (\%) & $\begin{array}{l}\text { Group A } \\
\mathrm{n}(\%)\end{array}$ & $\begin{array}{l}\text { Group B } \\
\mathrm{n}(\%)\end{array}$ & p-value \\
\hline Osteoporosis & $15(18.3)$ & $10(17.6)$ & $5(20)$ & 0.791 \\
Depression & $14(17.1)$ & $11(19.3)$ & $3(12)$ & 0.419
\end{tabular}

${ }^{a}$ Each patient may have more than one risk factors and comorbidities CV Cardiovascular, eGFR Estimated glomerular filtrate rate

powerful studies should adjust for possible confounders (including concomitant drugs and co-morbidities such as hypertension or cholesterol level and its variations), and using immune parameters to provide more specific and detailed information from the pathogenic point of view. Also, we need studies with a different design to assess whether sofosbuvir or $\mathrm{HCV}$ eradication (and possible immune effects related to this eradication) are implicated. For instance, one could compare cardiac function in patients with sustained virological response with respect to those without response, or cardiac function may be evaluated in healthy volunteers. Moreover, a group of control patients

Table 2 Prescribed treatment, supportive drugs and related outcome $(n=82)$

\begin{tabular}{ll}
\hline Cate & $\mathrm{n}(\%)$ \\
\hline Prescribed DAAs & \\
SOF + RBV & $10(12.2)$ \\
$\mathrm{SOF}+\mathrm{SIM} \pm \mathrm{RBV}$ & $29(35.4)$ \\
$\mathrm{SOF}+\mathrm{LDV} \pm$ RBV & $31(37.8)$ \\
SOF + DCV \pm RBV & $12(14.6)$ \\
Ribavirin & \\
Yes & $49(59.7)$ \\
No & $33(40.3)$ \\
Ribavirin modification & \\
None & $26(53.1)$ \\
Reduction & $22(44.9)$ \\
Suspension & $1(2)$
\end{tabular}

Adding support drug for anaemia in patients with ribavirin

None

38 (77.6)

Folic acid 7 (14.2)

Erythropoietin $2(4.1)$

Folic acid + erythropoietin 2 (4.1)

Reason for stopping DAAs

End of treatment $\quad 80$ (97.6)

Patient decision $1(1.2)$

Virological failure $1(1.2)$

DAAs Direct antiviral agents, SOF Sofosbuvir, RBV Ribavirin, SIM Simeprevir, LDV Ledipasvir, DCV Daclatasvir 


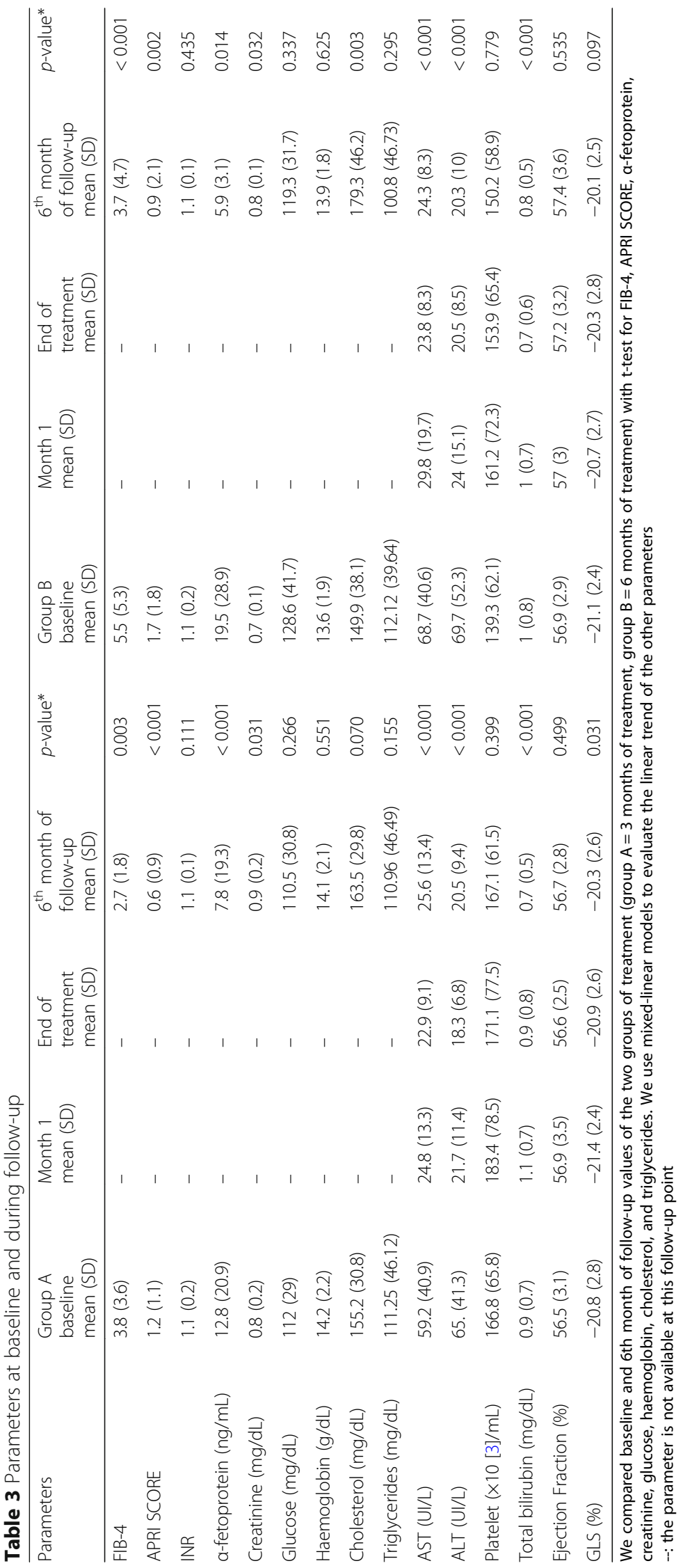




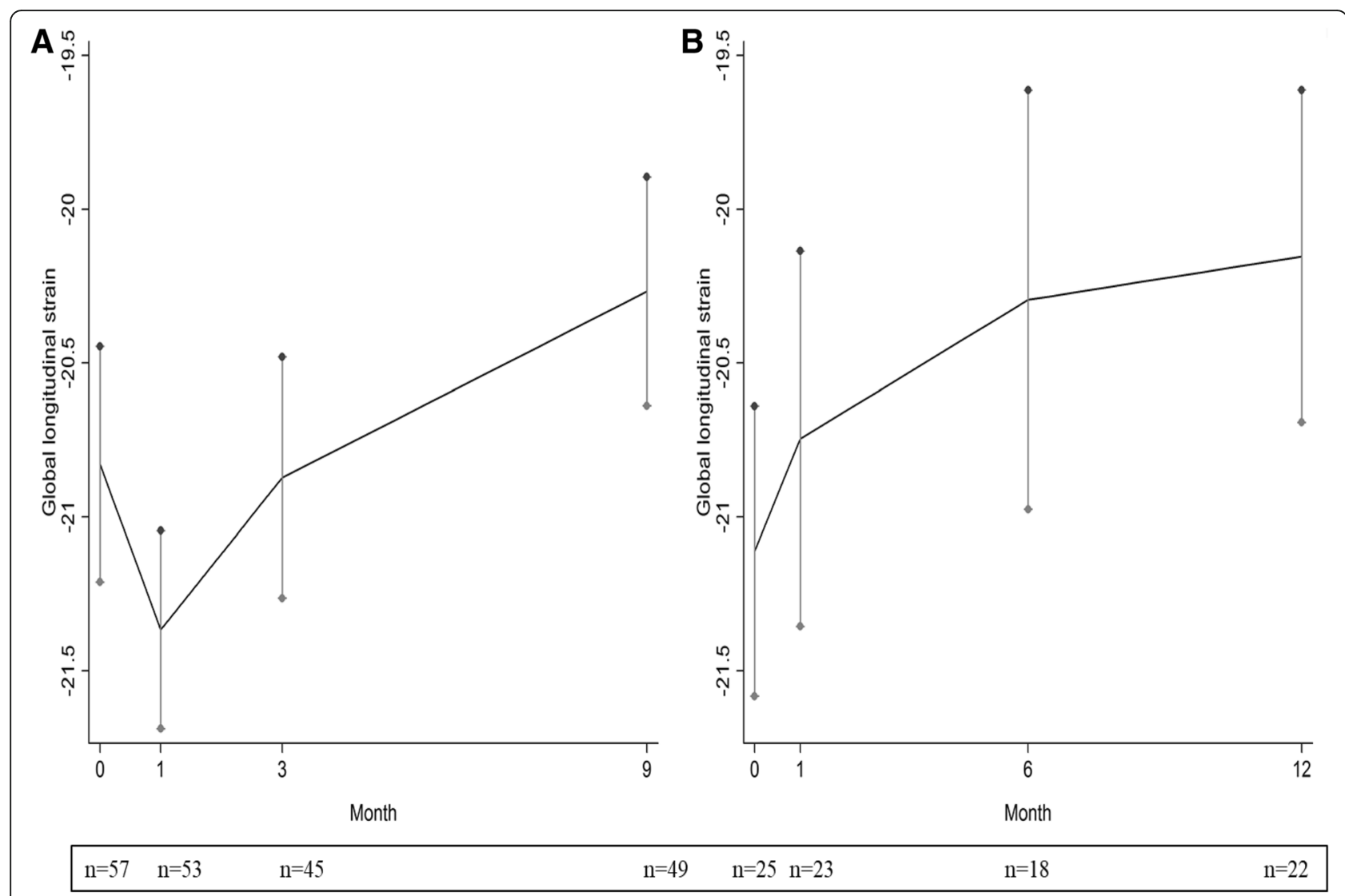

Fig. 2 Distribution of global longitudinal strain (GLS) at baseline, 1st month, at end of treatment and at 6th month of follow-up after treatment in the two groups of patients by treatment length, either 3 months (group A) or 6 months (group B). At each time point, the number of patient who performed cardiac ultrasound is showed below the $\mathrm{X}$-axis

with other aetiologies presenting the same risk factors, but not treated with sofosbuvir-based treatments would be helpful. Unfortunately, however, this is difficult (or unethical) to be accepted for the legitimate desires of patients to be treated as soon as possible.

Since we did not find any significant correlations between GLS and ribavirin or anaemia (data not shown), we may hypothesize that these factors were not implicated. However, we have to take into account that the small sample size reduced the power to detect a smaller effect of ribavirin, significantly. Indeed, the evidence of a prolonged worsening of GLS after stopping treatment is more consistent with an effect of ribavirin (whose multiple dose half-life is around 12 days, persisting in non-plasma compartments for as long as 6 months) than with an effect of sofosbuvir (whose half life is only $0.4 \mathrm{~h}$ ). For the same reason, the trend in GLS is more consistent with an immune-mediated phenomenon occurring after viral eradication, so consideration of immune markers could provide better insights on the phenomenon. With regard to hypertension, we did not find any significant evidences of a possible role at interaction model independently from time, but a complete assessment in a multivariable model would require grater numbers and a time-dependent consideration of hypertension as a variable in future studies.

In patients treated for three months, we noted an initial improvement of GLS, followed by a progressive worsening. The first phase of improvement could be due to a beneficial reduction of HCV RNA [10] while apparent sofosbuvir toxicity or other negative phenomena may have become more evident afterwards. This biphasic trend was not evident in patients treated for 6 months. The fact that patients who received 6 months of treatment were older, more likely to present advanced liver fibrosis or cirrhosis and comorbidities (including cardiac ones) could explain the discordant trends of GLS in the two groups. In fact, healthier individuals could benefit more from HCV RNA clearance in the short-term, while more compromised patients may suffer from a more prompt cardiotoxicity of sofosbuvir. Thus, it is worth considering that extreme elderly patients are receiving DAA treatments, with a high SVR rate, but at the same time they may experience more frequent cardiovascular complications, therefore a close and accurate monitoring of heart function could be required [36-38]. 
The associations between worse GLS and smoking or high BMI at baseline was not unexpected, suggesting the importance to quit negative behaviours, such as smoking and unhealthy diet in patients chronically infected by $\mathrm{HCV}$. This is even more relevant if one considers that cardiac function may worsen after treatment, concomitantly with an increase of cholesterol occurring after $\mathrm{HCV}$ eradication as demonstrated in our study and confirmed by others [39]. Appropriate time dependent analysis should be conducted to assess whether variations in cholesterol levels may lead to GLS changes during SOF-based regimens.

\section{Conclusions}

In conclusions, if confirmed by datasets from independent cohorts to replicate the data, our results are important because demonstrated for the first time the possible cardiotoxicity of DAA treatments. The same study protocol for patients who are eligible for DAAs treatment with sofosbuvir-free regimens should be applied, in order to evaluate whether worsening of GLS is a specific drug-related or a class effect. While these results should be confirmed in more powerful studies and pathogenic hypotheses should be tested in translational studies, in the meantime a cautious approach should include assessment of cardiac function during DAA treatment, particularly for the most fragile patients, who may benefit from interventions to reduce the risk of cardiovascular diseases both before and after treatment.

\section{Additional file}

Additional file 1: Table S1. AIFA (Italian Regulatory Agency for Drug Administration) criteria for prescription of a DAA treatment. Table S2. Association between global longitudinal strain (dichotomized on median) and demographical and clinical features at baseline.

Table S3. Multivariate mixed model, effect of time, BMI, presence of significant liver fibrosis and duration of treatment on global longitudinal strain. (DOCX $24 \mathrm{~kb}$ )

\section{Abbreviations \\ AIFA: Italian medicinal agency; APRI: AST to platelets ratio; BMI: Body mass index; CVD: Cardiovascular diseases; DAAs: Direct antiviral agents; DCV: Daclatasvir; EF: Ejection fraction; eGFR: Estimated glomerular filtrate rate; FIB 4: Fibrosis 4 index; GLS: Global longitudinal strain; HCV: Hepatitis C virus; LDV: Ledipasvir; LVF: Left ventricular function; RBV: Ribavirin; SD: Standard deviation; SIM: Simeprevir; SOF: Sofosbuvir; SVR: Sustained virological response}

\section{Acknowledgements}

We want to thank all the patients who accepted to participate in our study. The CARDIAC Study group at UMG includes: Giorgio Settimo Barreca, Francesco Saverio Costanzo, Daniela Foti, Giorgio Fuiano, Giuseppe Greco, Francesca Serapide, Elio Gulletta, Nadia Marascio, Maria Concetta Postorino, Maria Adelina Simeoni, Alfredo Focà, Maria Carla Liberto, Aida Giancotti. This work has been presented in part at the American Association for the Study of Liver Diseases (AASLD) - The Liver Meeting ${ }^{\circledR}$ 2017, October 20-24 2017, Washington DC (poster \#1079).
Funding

CARDIAC Study (Cardiovascular Diseases in new Antiviral Therapies for HCV) did not receive specific grant from any funding agency in the public, commercial and non-profit sectors.

Availability of data and materials

All data of this study are included in the present manuscript.

\section{Authors' contributions}

MM designed the study, wrote the protocol, acquired informed consent, collected and processed the data, and contributed to write the manuscript; $\mathrm{CT}$ coordinated the protocol, and contributed to write and revise the final version of the manuscript; JS contributed in clinical management of patients, performed cardiac ultrasound, and helped in data collection and in the revision of final version of the manuscript; GLDA performed cardiac ultrasound and helped in data collection; CC contributed in clinical management of patients and in data collection; VP contributed in clinical management of patients and in data collection; ER processed data and performed all statistical analysis; AS contributed in clinical management of patients and in data collection; SDR contributed to write the manuscript and helped in the revision of final version; AF coordinated microbiological tests, helped to revise the final version of the manuscript; $M C L$ coordinated microbiological tests, helped to revise the final version of the manuscript; $\mathrm{Cl}$ coordinated the protocol, contributed to write and to revise the final version of the manuscript. All the authors read and approved the final version of the manuscript

\section{Ethics approval and consent to participate}

This study was conducted in accordance with the guidelines of the Declaration of Helsinki and the principles of Good Clinical Practice. The local Ethical Committee (Calabria Region) approved the study protocol on March 13th, 2015, and written informed consent was obtained from all subjects enrolled.

\section{Consent for publication}

Not applicable.

\section{Competing interests}

The authors declare that they have no competing interests..

\section{Publisher's Note}

Springer Nature remains neutral with regard to jurisdictional claims in published maps and institutional affiliations.

\section{Author details}

${ }^{1}$ Unit of Infectious and Tropical Diseases, Department of Medical and Surgical Sciences, "Magna Graecia" University of Catanzaro, Viale Europa, 88100 Catanzaro, Italy. ${ }^{2}$ Cardiovascular Institute, Department of Medical and Surgical Sciences, "Magna Graecia" University of Catanzaro, Viale Europa, 88100 Catanzaro, Italy. ${ }^{3}$ Unit of Hygiene, Epidemiology and Public Health, Department of Medical and Surgical Specialities, Radiological Sciences and Public Health, Viale Europa, 25123 Brescia, Italy. ${ }^{4}$ Institute of Microbiology, Department of Health Sciences, "Magna Graecia" University of Catanzaro, Viale Europa, 88100 Catanzaro, Italy.

Received: 21 May 2018 Accepted: 1 October 2018

Published online: 16 October 2018

References

1. Ferri C, Sebastiani M, Giuggioli D, et al. Hepatitis C virus syndrome: a constellation of organ- and non-organ specific autoimmune disorders, B-cell non-Hodgkin's lymphoma, and cancer. World J Hepatol. 2015;7(3):327-43.

2. Gill K, Ghazinian H, Manch R, Gish R. Hepatitis C virus as a systemic disease: reaching beyond the liver. Hepatol Int. 2016;10(3):415-23.

3. Ambrosino P, Lupoli R, Di Minno A, et al. The risk of coronary artery disease and cerebrovascular disease in patients with hepatitis C: a systematic review and meta-analysis. Int J Cardiol. 2016;221:746-54.

4. Zampino R, Marrone A, Restivo L, et al. Chronic HCV infection and inflammation: clinical impact on hepatic and extra-hepatic manifestations. World J Hepatol. 2013;5(10):528-40. 
5. Adinolfi LE, Zampino R, Restivo L, et al. Chronic hepatitis C virus infection and atherosclerosis: clinical impact and mechanisms. World J Gastroenterol. 2014;20(13):3410-7.

6. Matsumori A. Role of hepatitis C virus in cardiomyopathies. Ernst Schering Res Found Workshop. 2006;55:99-120.

7. Matsumori A, Shimada T, Chapman NM, Tracy SM, Mason JW. Myocarditis and heart failure associated with hepatitis C virus infection. J Card Fail. 2006; 12(4):293-8.

8. Matsumori A, Yutani C, Ikeda Y, Kawai S, Sasayama S. Hepatitis C virus from the hearts of patients with myocarditis and cardiomyopathy. Lab Investig. 2000;80(7):1137-42.

9. SOVALDI ${ }^{\oplus}$ (sofosbuvir) tablets, for oral use initial U.S. 2013. Published 2013. https://www.accessdata.fda.gov/drugsatfda_docs/label/2015/ 204671s004lbl.pdf. Accessed 7 Oct 2018.

10. Hsu YC, Lin JT, Ho HJ, et al. Antiviral treatment for hepatitis C virus infection is associated with improved renal and cardiovascular outcomes in diabetic patients. Hepatology. 2014:59(4):1293-302.

11. Nahon P, Bourcier V, Layese R, et al. Eradication of Hepatitis C Virus Infection in Patients With Cirrhosis Reduces Risk of Liver and Non-Liver Complications. Gastroenterology. 2017;152(1):142-156.e142.

12. Folland ED, Parisi AF, Moynihan PF, Jones DR, Feldman CL, Tow DE. Assessment of left ventricular ejection fraction and volumes by real-time, two-dimensional echocardiography. A comparison of cineangiographic and radionuclide techniques. Circulation. 1979;60(4):760-6.

13. Marcucci CE, Samad Z, Rivera J, et al. A comparative evaluation of transesophageal and transthoracic echocardiography for measurement of left ventricular systolic strain using speckle tracking. J Cardiothorac Vasc Anesth. 2012;26(1):17-25.

14. Reisner SA, Lysyansky P, Agmon Y, Mutlak D, Lessick J, Friedman Z. Global longitudinal strain: a novel index of left ventricular systolic function. J Am Soc Echocardiogr. 2004;17(6):630-3.

15. Sengeløv M, Jørgensen PG, Jensen JS, et al. Global longitudinal strain is a superior predictor of all-cause mortality in heart failure with reduced ejection fraction. JACC Cardiovasc Imaging. 2015:8(12):1351-9.

16. Piegari E, Di Salvo G, Castaldi B, et al. Myocardial strain analysis in a doxorubicininduced cardiomyopathy model. Ultrasound Med Biol. 2008;34(3):370-8.

17. Thavendiranathan P, Poulin F, Lim KD, Plana JC, Woo A, Marwick TH. Use of myocardial strain imaging by echocardiography for the early detection of cardiotoxicity in patients during and after cancer chemotherapy: a systematic review. J Am Coll Cardiol. 2014;63(25 Pt A):2751-68.

18. Sims A, Frank L, Cross R, et al. Abnormal cardiac strain in children and young adults with HIV acquired in early life. J Am Soc Echocardiogr. 2012; 25(7):741-8

19. Kasner $M$, Sinning $D$, Escher $F$, et al. The utility of speckle tracking imaging in the diagnostic of acute myocarditis, as proven by endomyocardial biopsy. Int J Cardiol. 2013;168(3):3023-4.

20. Khosla T, Lowe CR. Indices of obesity derived from body weight and height. Br J Prev Soc Med. 1967;21(3):122-8.

21. Adler M, Gulbis B, Moreno C, et al. The predictive value of FIB-4 versus FibroTest, APRI, Fibrolndex and Forns index to noninvasively estimate fibrosis in hepatitis $C$ and nonhepatitis $C$ liver diseases. Hepatology. 2008; 47(2):762-3 author reply 763

22. Bota S, Sirli R, Sporea I, et al. A new scoring system for prediction of fibrosis in chronic hepatitis C. Hepat Mon. 2011;11(7):548-55.

23. Vallet-Pichard A, Mallet $V$, Nalpas B, et al. FIB-4: an inexpensive and accurate marker of fibrosis in HCV infection. Comparison with liver biopsy and fibrotest. Hepatology. 2007:46(1):32-6.

24. http://www.hep-druginteractions.org. Accessed 8 Oct 2018

25. Vijayananthan A, Nawawi $\mathrm{O}$. The importance of good clinical practice guidelines and its role in clinical trials. Biomed Imaging Interv J. 2008;4(1):e5.

26. Ndebele $P$. The declaration of Helsinki, 50 years later. JAMA. 2013;310(20): 2145-6.

27. Association WM. World medical association declaration of Helsinki: ethical principles for medical research involving human subjects. JAMA. 2013; 310(20):2191-4.

28. Yingchoncharoen T, Agarwal S, Popović ZB, Marwick TH. Normal ranges of left ventricular strain: a meta-analysis. J Am Soc Echocardiogr. 2013;26(2):185-91.

29. Marwick TH, Leano RL, Brown J, et al. Myocardial strain measurement with 2-dimensional speckle-tracking echocardiography: definition of normal range. JACC Cardiovasc Imaging. 2009;2(1):80-4.
30. Ahmad T, Yin P, Saffitz J, et al. Cardiac dysfunction associated with a nucleotide polymerase inhibitor for treatment of hepatitis C. Hepatology. 2015;62(2):409-16.

31. Babuin L, Jaffe AS. Troponin: the biomarker of choice for the detection of cardiac injury. CMAJ. 2005;173(10):1191-202.

32. Vuolteenaho O, Ala-Kopsala M, Ruskoaho H. BNP as a biomarker in heart disease. Adv Clin Chem. 2005:40:1-36.

33. Divakaran $\mathrm{V}$, Mann DL. The emerging role of microRNAs in cardiac remodeling and heart failure. Circ Res. 2008;103(10):1072-83.

34. Corsten MF, Dennert R, Jochems S, et al. Circulating MicroRNA-208b and MicroRNA-499 reflect myocardial damage in cardiovascular disease. Circ Cardiovasc Genet. 2010;3(6):499-506.

35. Burdette $D$, Haskett A, Presser L, McRae S, labal J, Waris G. Hepatitis C virus activates interleukin-1 $\beta$ via caspase-1-inflammasome complex. J Gen Virol. 2012;93(Pt 2):235-46.

36. Toyoda H, Kumada T, Tada T, et al. Efficacy and tolerability of an IFN-free regimen with DCV/ASV for elderly patients infected with HCV genotype 1B. J Hepatol. 2017:66(3):521-7.

37. Ji F, Wei B, Yeo YH, et al. Systematic review with meta-analysis: effectiveness and tolerability of interferon-free direct-acting antiviral regimens for chronic hepatitis $C$ genotype 1 in routine clinical practice in Asia. Aliment Pharmacol Ther. 2018:47(5):550-62

38. Ji F, Tian C, Li Z, Deng H, Nguyen MH. Ledipasvir and sofosbuvir combination for hepatitis $C$ virus infection in three patients aged 85 years and older. Eur J Gastroenterol Hepatol. 2017;29(8):977-9.

39. Mauss $\mathrm{S}$, Berger $\mathrm{F}$, Wehmeyer $\mathrm{MH}$, et al. Effect of antiviral therapy for HCV on lipid levels. Antivir Ther. 2017;21(1):81-8.

\section{Ready to submit your research? Choose BMC and benefit from:}

- fast, convenient online submission

- thorough peer review by experienced researchers in your field

- rapid publication on acceptance

- support for research data, including large and complex data types

- gold Open Access which fosters wider collaboration and increased citations

- maximum visibility for your research: over $100 \mathrm{M}$ website views per year

At BMC, research is always in progress.

Learn more biomedcentral.com/submissions 